University of Nebraska - Lincoln

DigitalCommons@University of Nebraska - Lincoln

\title{
Venous Thromboembolic Disease in Trauma and Surveillance Ultrasonography
}

\author{
Randeep S. Jawa \\ Kathy Warren \\ David Young \\ Michael Wagner \\ Lawrence Nelson
}

See next page for additional authors

Follow this and additional works at: https://digitalcommons.unl.edu/usafresearch

This Article is brought to you for free and open access by the U.S. Department of Defense at

DigitalCommons@University of Nebraska - Lincoln. It has been accepted for inclusion in U.S. Air Force Research by an authorized administrator of DigitalCommons@University of Nebraska - Lincoln. 


\section{Authors}

Randeep S. Jawa, Kathy Warren, David Young, Michael Wagner, Lawrence Nelson, Diane Yetter, Shane Banks, Valerie Shostrom, and Joseph Stothert 


\title{
ASSOCIATION FOR ACADEMIC SURGERY
}

\section{Venous Thromboembolic Disease in Trauma and Surveillance Ultrasonography ${ }^{1}$}

\author{
Randeep S. Jawa, M.D., ${ }^{* 2}$ Kathy Warren, R.N.,† David Young, M.D., ${ }^{*}$ Michel Wagner, M.D., ${ }^{*}$ \\ Lawrence Nelson, M.D., ${ }^{*}$ Diane Yetter, R.N.,† Shane Banks, M.D.,§ Valerie Shostrom, M.S.,末 \\ and Joseph Stothert, M.D.* \\ *Department of Surgery, University of Nebraska Medical Center, Omaha, Nebraska; $\uparrow$ Nebraska Medical Center, Omaha, Nebraska; \\ $\ddagger$ College of Public Health, University of Nebraska Medical Center, Omaha, Nebraska; and §United States Air Force
}

Submitted for publication March 29, 2010

\begin{abstract}
Background. The literature reports a wide variation in the incidence of venous thromboembolic (VTE) disease in trauma patients. The performance of routine surveillance venous duplex ultrasound of bilateral lower extremities is controversial. Furthermore, recent examinations of the national trauma databank registry have suggested that routine duplex surveillance is associated with higher deep venous thrombosis (DVT) detection rates.

Materials and Methods. We examined the incidence and risk factors for VTE disease in 2827 trauma patients admitted over a $2-y$ period to a state-verified level I trauma center. Detailed chart review was carried out for patients with VTE disease. We then evaluated the effects of a routine bilateral lower extremity duplex surveillance guideline on VTE detection in the subset of injury patients admitted to the trauma service.
\end{abstract}

Results. We found an approximately $2 \%$ incidence of venous thromboembolic disease in a mostly blunt trauma population. Amongst patients with VTE disease, the most common risk factors were obesity and significant head injury. We then evaluated the 998 patients with injury who were admitted to the trauma service 1 y before and after surveillance guideline implementation. Despite a nearly 5 -fold increase in the number of duplex scans, with a substantial increase in cost, we found no significant difference in the incidence of DVT.

Conclusions. Our preliminary data argue against the use of routine duplex surveillance of lower extrem-

\footnotetext{
${ }^{1}$ Presented in part at the 5th Annual Academic Surgical Congress in San Antonio, Texas, February 2010.

${ }^{2}$ To whom correspondence and reprint requests should be addressed at Department of Surgery, UNMC, 983280 Nebraska Medical Center, Omaha, NE 68198-3280. E-mail: rjawa@unmc.edu.
}

ities for DVT in trauma patients. A larger, prospective analysis is necessary to confirm these findings. (๑) 2011 Elsevier Inc. All rights reserved.

Key Words: trauma; DVT; PE; thrombus; venous; pulmonary; embolism; duplex; ultrasound; surveillance.

\section{INTRODUCTION}

Trauma is a risk factor for venous thromboembolic (VTE) disease. The literature suggests a relatively high incidence of VTE disease in trauma patients. However, there is lack of consensus on the exact incidence of VTE disease in trauma patients, and on the best method of VTE prevention [1]. Rates of deep venous thrombosis (DVT) have been reported to range from $0.36 \%$ to $90 \%$ in trauma patients [2-6]. Reasons for this wide range are myriad, but include the inclusion or exclusion of infrageniculate thrombi and thromboprophylaxis method used.

As trauma and critically ill patients are considered to be at high-risk for DVT and its clinical diagnosis is problematic, many centers have implemented routine duplex surveillance programs of lower extremity veins. It has been suggested that because greater than $50 \%$ of patients with typical DVT symptoms do not have DVT and conversely greater than $66 \%$ of significant DVT are not clinically recognized, imaging should be routinely performed for DVT detection in surgical intensive care unit patients [6]. To this end, as a level III practice management guideline, the Eastern Association for the Surgery of Trauma (EAST) suggested that serial duplex ultrasound scanning of high-risk asymptomatic trauma patients may be cost-effective and decrease the incidence of pulmonary embolism (PE) [7]. 
Similarly, in 2004, an American College of Chest Physicians (ACCP) consensus conference recommended surveillance duplex scans only in high-risk trauma patients who are unable to receive prophylaxis [8]. In a 2008 update, as a grade $1 \mathrm{C}$ recommendation, the ACCP recommended screening duplex exams in highrisk patients (i.e., spinal cord injury, lower-extremity or pelvic fracture, or major head injury) who have received suboptimal or no thromboprophylaxis [9]. However, as a grade $1 \mathrm{~B}$ recommendation, they advised against routine duplex screening for asymptomatic DVT in trauma patients [9].

In turn, evaluation of the National Trauma Databank (NTDB) registry data demonstrated that increased surveillance results in increased DVT detection rates. In 2008, Pierce et al. [5] interrogated the NTDB ver. 6.1 registry data and concluded that hospitals with more aggressive lower extremity DVT screening procedures had higher DVT rates. They found that in general, every percentage increase in surveillance rate increased the reported DVT rate by $7 \%$ [5]. In 2009, these authors analyzed ver. 6.2 of the National Trauma Data Bank and found that admission to a hospital that routinely screened for DVT was an independent predictor of DVT diagnosis, after controlling for risk factors [10]. Huseynova et al. on evaluation of ver. 7.1 of the National Trauma Data Bank came to a similar conclusion [2].

In November 2006, the trauma service at our stateverified level one trauma center implemented a guideline of routinely performing surveillance duplex scans of bilateral lower extremities in high-risk trauma patients. Given the recent literature demonstrating increased lower extremity DVT detection rates with routine duplex surveillance, we hypothesized that the incidence of lower extremity DVT after implementation of this guideline would increase. Additional specific aims of this study were to elucidate the incidence of VTE events in trauma patients admitted to our hospital, regardless of admitting service, and to examine the incidence of commonly described risk factors in trauma patients with VTE events.

\section{METHODS}

\section{Data Collection and Analysis}

After obtaining University of Nebraska Medical Center Institutional Review Board approval, we retrospectively queried our trauma registry. This registry, which is captured by the NTRACS ver. 4 database (Digital Innovations, Forest Hill, MD), includes all patients with injury admitted to the hospital. The minimum length of stay in the registry is $1 \mathrm{~d}$, which equates with a stay of up to $24 \mathrm{~h}$. Individual charts of patients with venous thromboembolic events were subsequently examined for comorbid conditions and injury details. In tabulating DVT and PE data, only patients with imaging-proven venous thromboembolic disease were counted. In calculation of statistical values, data were generally rounded to the nearest integer. To provide uniformity to length of stay measurement in patients with VTE, for patients transferred to our trauma center from an outside facility, hospital length of stay included the length of stay at the referring hospital plus the length of stay at our institution. For definition purposes, suprageniculate DVT included popliteal vein DVT. Statistical analysis was carried out using PC SAS ver. 9.2 (SAS, Cary, NC), in consultation with the Biostatistics Department of the College of Public Health, University of Nebraska Medical Center. A $t$-test was used for continuous data and the $\chi^{2}$ test was used for categorical data. Fisher's exact test was also used in comparing the percentage of patients admitted to the trauma service with VTE, before and after implementation of the guideline. A test was considered statistically significant if $P \leq 0.05$.

\section{Routine Lower Extremity Venous Duplex Ultrasound Surveillance Protocol}

In November 2006, we initiated a guideline whereby high-risk trauma patients admitted to the trauma service, with an expected hospital length of stay $>2 \mathrm{~d}$, undergo routine duplex ultrasound surveillance of bilateral lower extremities for venous thrombosis. Criteria for identifying high-risk trauma patients include age $>55$ $\mathrm{y}$, expected immobilization $>3 \mathrm{~d}$, multiple blood transfusions, surgery lasting longer than $2 \mathrm{~h}$, severe head injury, spinal cord injury, major venous injury, complicated pelvic fracture, and lower extremity fracture. Additional criteria for high-risk trauma patients include previous DVT history, obesity, hormone therapy, and current or recent pregnancy. These criteria mirror high-risk criteria identified in the literature [11-13]. This protocol advised that screening should be performed on high-risk patients within the first 2 to $3 \mathrm{~d}$ of admission. All trauma service admissions after November 2006 were subject to the above guideline, however, given the above recommendations, the definition of high-risk, and therefore the decision to implement lower extremity surveillance venous ultrasonography was left to the physician's discretion. After the initial screening, weekly ultrasound examinations were done to monitor current DVT status or to continue surveillance in the event of a negative scan. Our protocol has similarities to other surveillance programs, where an initial scan is performed early in high-risk trauma patients, followed by serial surveillance scans $[4,11]$. Duplex scanning was performed using linear $(3-11 \mathrm{MHz})$ or sector $(3-4 \mathrm{MHz})$ scan heads. The pelvic veins were not assessed. Data on iliac vein thromboses was gathered separately by computerized tomography (CT) scan and/ or ultrasound. DVT prophylaxis standing orders at admission to the trauma service include options for sequential compression devices, subcutaneous heparin (5000 units subcutaneously three times daily), enoxaparin (30 mg subcutaneously twice daily or $40 \mathrm{mg}$ once daily), or no DVT prophylaxis in patients who are ambulating normally. Mechanical and chemical prophylaxis are not mutually exclusive.

\section{RESULTS}

In querying our trauma registry, we found that in the 12 mo preceding initiation of routine bilateral lower extremity duplex ultrasound surveillance scanning for DVT (late 2005-late 2006), there were 1342 patients admitted for injury to various services of the hospital. In the 12 mo following initiation of routine surveillance (late 2006-late 2007), there were 1485 patients admitted for injury to several different hospital services. In examining the demographics of the trauma population in late 2005-2006 and late 2006-2007, we noted comparable mean age ( $49 \mathrm{y}$ and $48 \mathrm{y}$ ), gender, hospital length 
of stay, percent of patients with blunt trauma (95\% and 94\%), and injury severity score (ISS 11 and 11), $P$ not significant (Table 1).

Approximately $1.9 \%$ (55 patients) of the 2827 patients admitted for injury developed venous thromboembolic events (DVT and/or PE), where $1.6 \%$ of patients (46) experienced DVT with or without PE (Table 2). Approximately $11 \%$ of 46 patients with DVT also had a PE. Only $19 \%$ of these 46 patients had symptomatic DVT. Lower extremity DVT occurred on average on postinjury $\mathrm{d} 11$, with a standard deviation of $12 \mathrm{~d}$, and a range of 0 to $58 \mathrm{~d}$ postinjury. Of note, approximately $13 \%$ of the 46 patients with DVT were noted to have DVT by postinjury day one; with one DVT being noted on day of injury. In examining the demographic characteristics of the 55 patients admitted with VTE, regardless of admitting service, the most common findings were blunt trauma in $93 \%$, obesity with a body mass index $(\mathrm{BMI})>30$ in $44 \%$, significant head injury (AIS $\geq 3$ ) in $31 \%$, mechanical ventilation for 3 or more d in $27 \%$, surgery $>2 \mathrm{~h}$ in $27 \%$, two or more long bone extremity fractures/dislocations in 24\%, and transfusion of 4 or more units of PRBC in 24\%. PRBC and FFP were administered at various time points as needed, i.e., during hospitalization, during resuscitation, intraoperatively, and as needed to reverse injury or coagulopathy secondary to prior anticoagulant use. Specifically, with regards to PRBC use, five patients received uncrossmatched blood as part of their resuscitation, with four of these patients undergoing surgery on day of admission. Furthermore, in evaluating significant past medical history that would increase VTE risk, nine patients with VTE also had a history of cancer, DVT, or hormonal therapy. One additional patient was found to have Factor II deficiency.

We noted that the majority (28) of patients had infrageniculate only DVT, six patients had only suprageniculate DVT (including one who also had iliac vein thrombus), 12 patients had combined supra- and infrageniculate DVT (including one who also had iliac vein

\section{TABLE 1}

\section{Demographics of Total Injury Population}

\begin{tabular}{lcc}
\hline & $2005-2006$ & $2006-2007$ \\
\hline $\begin{array}{l}\text { Patients with injury } \\
\text { Average age } \pm \mathrm{SD} \\
\quad \text { (range) }\end{array}$ & $49 \pm 25 \mathrm{y}(0-102 \mathrm{y})$ & $48 \pm 26 \mathrm{y}(0-100 \mathrm{y})$ \\
$\begin{array}{l}\text { Percent male } \\
\text { Average hospital } \\
\text { length of stay } \pm \mathrm{SD} \\
\quad \text { (range) }\end{array}$ & 56 & 57 \\
$\begin{array}{l}\text { Average ISS } \pm \text { SD (range) } \\
\text { Percent blunt }\end{array}$ & $10 \pm 10(1-75)$ & $10 \pm 10(1-75)$ \\
\hline
\end{tabular}

thrombus). With regards to prophylaxis, 32/46 (68\%) patients with DVT were receiving chemical prophylaxis on the day of or on the day immediately prior to DVT diagnosis; 15 of these patients were receiving enoxaparin. Nine of 14 patients with pulmonary embolism with or without DVT were receiving chemical prophylaxis; seven were receiving enoxaparin. With regards to management of VTE events, $41 \%$ of patients had IVC filters placed. However, this was not mutually exclusive with therapeutic anticoagulation, as patients were also therapeutically anticoagulated when possible. Specifically, $17 / 22$ patients eventually received therapeutic anticoagulation as well as an IVC filter

As the routine lower extremity duplex surveillance protocol was implemented only in injured patients who were admitted to the trauma service, we evaluated this subset of patients for the $1 \mathrm{y}$ before (late 2005-late 2006) and 1 y after (late 2006-late 2007) guideline initiation. Nine-hundred ninety-eight patients had a total of 1017 admissions to the trauma service; some of these patients were admitted more than once (Table 3 ). The 459 patients who were admitted to the trauma service before surveillance guideline implementation were significantly younger, more severely injured, and had longer hospital length of stay than the 1342 patients who were admitted to all services of the hospital over the same time frame. Similarly, the 539 patients admitted to the trauma service after guideline implementation were also significantly younger and more severely injured than the 1485 patients admitted to all hospital services with injury over the same time frame. However, their length of stay was not significantly different.

Venous thromboembolic disease was present in a minority of patients with injury admitted to the trauma service, with a prevalence of $12 / 459$ (2.6\%) prior to implementation of routine duplex surveillance and 15/ $539(2.8 \%)$ after initiation of routine ultrasound surveillance (Table 3). This difference was not statistically significant $(P=0.99)$, as evaluated by Fisher's exact test. Of note, three DVT events occurred in children. The first patient, in the prescreen group, was a blunt trauma patient with head injury, who had an asymptomatic DVT. The other two patients had penetrating major vascular injury (iliac vein and femoral vein, respectively). The patient with iliac vein injury also had an asymptomatic DVT. Of note, two of the patients with DVT were admitted before guideline implementation, but their scans were performed in the time period following guideline implementation. If the data is tabulated in this manner, there were 10 patients with DVT of $457(2.2 \%)$ pre-guideline trauma service patients and 16 patients with DVT in 541 (3.0\%) post-guideline patients. However, the event rate remains statistically nonsignificant, $P=0.55$. 
TABLE 2

VTE Patient Demographics

\begin{tabular}{|c|c|c|c|c|c|}
\hline & $\begin{array}{l}2005-2006 \\
\text { DVT alone }\end{array}$ & $\begin{array}{l}2005-2006 \\
P E \pm D V T\end{array}$ & $\begin{array}{l}2006-2007 \\
\text { DVT alone }\end{array}$ & $\begin{array}{c}2006-2007 \\
P E \pm D V T\end{array}$ & $\begin{array}{c}\text { Total } \\
\text { (\% of total VTE) }\end{array}$ \\
\hline Patients $(\mathrm{N}, \%)$ & 24 & $7(0.5 \%)(4$ had both $)$ & $17(1.1)$ & $7(0.5 \%)$ (1 had both) & $55(100 \%)$ \\
\hline Average age (range) & $50 \pm 19(12-88)$ & $75 \pm 13(53-90)$ & $53 \pm 22(11-91)$ & $68 \pm 16(43-89)$ & \\
\hline Average ISS (range) & $23 \pm 16(1-59)$ & $12 \pm 6(9-25)$ & $20 \pm 9(5-38)$ & $14 \pm 11(4-38)$ & \\
\hline Average hospital LOS (range) & $22 \pm 16(4-65)$ & $13 \pm 14(2-44)$ & $17 \pm 14(2-44)$ & $17 \pm 9(7-34)$ & \\
\hline Blunt trauma $(\%)$ & $23(96 \%)$ & $7(100 \%)$ & $14(82 \%)$ & $7(100 \%)$ & $51(93 \%)$ \\
\hline Head injury $($ AIS > 3) $(\mathrm{N})$ & 8 & 2 & 6 & 1 & $17(31 \%)$ \\
\hline Spinal cord injury $(\mathrm{N})$ & 1 & 0 & 3 & 0 & $4(7 \%)$ \\
\hline Pelvic fracture $(\mathrm{N})$ & 3 & 0 & 1 & 1 & $5(9 \%)$ \\
\hline$\geq 2$ Extremity fractures/dislocations $(\mathrm{N})$ & 7 & 1 & 4 & 1 & $13(24 \%)$ \\
\hline 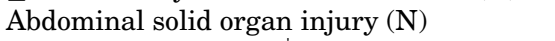 & 3 & 0 & 0 & 0 & $3(5 \%)$ \\
\hline Major vascular injury ${ }^{\dagger}$ & 1 (aorta) & 0 & $\begin{array}{l}2(1 \text { femoral a/v. } \\
1 \text { bilat. Iliac v. })\end{array}$ & 0 & $3(5 \%)$ \\
\hline Shock $(\mathrm{SBP}<90)$ at initial admission $(\mathrm{N})^{*}$ & 3 & 0 & 3 & 0 & $6(12 \%)$ \\
\hline Surgery $>2 \mathrm{~h}$ prior to VTE $(\mathrm{N})^{*}$ & 8 & 1 & 4 & 1 & $14(27 \%)$ \\
\hline Transfusion $\geq 4$ units PRBC $(\mathrm{N})^{*}$ & 9 & 1 & 3 & 0 & $13(24 \%)$ \\
\hline FFP transfusion $(\mathrm{N})^{*}$ & 3 & 0 & 3 & 0 & $6(11 \%)$ \\
\hline Factor VII use (N) & 1 & 0 & 0 & 0 & $1(2 \%)$ \\
\hline Mech. ventilation $\geq 3 \mathrm{~d}$ prior to VTE & 10 & 1 & 3 & 0 & $15(27 \%)$ \\
\hline $\mathrm{BMI}>30(\mathrm{~N}) * \mathrm{BMI}$ range & $12(17-53)$ & $1(17-31)$ & $7(19-42)$ & $3(22-38)$ & $24(44 \%)$ \\
\hline IVC filter placed $(\mathrm{N})$ & 11 & 3 & 7 & 1 & $22(40 \%)$ \\
\hline
\end{tabular}

* In calculating the percent of patients with these characteristics who developed VTE events, the denominator was reduced from 56 patients to exclude patients for whom the relevant data were not available. Specifically, the denominator was 48 for shock, 52 for surgery greater than 2 h, 53 for packed red blood cell (PRBC) transfusion, 53 for fresh frozen plasma (FFP) transfusion, and 54 for body mass index (BMI) calculation.

'In the three patients with major vascular injury, there was 1 aortic injury, 1 femoral artery and vein injury, and 1 bilateral iliac vein injury. 


\section{TABLE 3}

Patients Admitted to the Trauma Service

\begin{tabular}{|c|c|c|}
\hline & $\begin{array}{l}\text { Before surveillance } \\
\text { (N) }(2005-2006)\end{array}$ & $\begin{array}{l}\text { After surveillance } \\
\text { (N) (2006-2007) }\end{array}$ \\
\hline Trauma patients & 459 & 539 \\
\hline $\begin{array}{l}\text { Average LOS } \pm \text { SD } \\
\quad \text { (range) }\end{array}$ & $6 \pm 9(0-68)$ & $6 \pm 8(1-75)$ \\
\hline $\begin{array}{l}\text { Average age } \pm \mathrm{SD} \\
\quad(\text { range })\end{array}$ & $37 \pm 19(0.5-89)$ & $36 \pm 20(-92)$ \\
\hline $\begin{array}{l}\text { Average ISS } \pm \mathrm{SD} \\
\quad \text { (range) }\end{array}$ & $15 \pm 13(1-75)$ & $15 \pm 13(1-75)$ \\
\hline $\begin{array}{l}\text { Number of patients } \\
\text { with duplex scans }\end{array}$ & 40 & 133 \\
\hline Number of duplex scans & 54 & 253 \\
\hline $\begin{array}{l}\text { Mean (SD) number of } \\
\text { duplex scan per } \\
\text { scanned patient }\end{array}$ & $1.3 \pm 0.8$ & $1.9 \pm 1.7$ \\
\hline $\begin{array}{l}\text { Range of duplex scans } \\
\text { per scanned patient }\end{array}$ & $1-5$ & $1-12$ \\
\hline $\begin{array}{l}\text { Trauma admissions with } \\
\text { LOS }>2 \mathrm{~d}\end{array}$ & 248 & 284 \\
\hline $\begin{array}{l}\text { Average LOS } \pm \mathrm{SD} \\
\quad(\text { range of } d)\end{array}$ & $10 \pm 10(3-68)$ & $10 \pm 9(3-75)$ \\
\hline $\begin{array}{l}\text { Average age } \pm \mathrm{SD} \\
\quad(\text { range })\end{array}$ & $40 \pm 20(0.5-89)$ & $41 \pm 21(1-92)$ \\
\hline $\begin{array}{l}\text { Average ISS } \pm \mathrm{SD} \\
\quad \text { (range) }\end{array}$ & $20 \pm 13(1-66)$ & $20 \pm 13(-75)$ \\
\hline $\begin{array}{l}\text { Number of patients } \\
\text { with duplex scans }\end{array}$ & 40 & 122 \\
\hline $\begin{array}{l}\text { Total trauma patients } \\
\text { with VTE disease }\end{array}$ & 12 & 15 \\
\hline $\begin{array}{l}\text { Average age } \pm \text { SD } \\
\quad \text { (range) }\end{array}$ & $44 \pm 16(12-61)$ & $50 \pm 20(11-70)$ \\
\hline $\begin{array}{l}\text { Average ISS } \pm \mathrm{SD} \\
\quad(\text { range })\end{array}$ & $35 \pm 12(18-45)$ & $34 \pm 8(9-34)$ \\
\hline Patients with DVT & 12 & 14 \\
\hline Symptomatic DVT & 1 & 2 \\
\hline Patients with PE & 0 & 1 \\
\hline Number of duplex scans & 37 & 52 \\
\hline
\end{tabular}

We found that the number of duplex scans increased by 4.7 times, from 54 to 253 after guideline implementation, supporting the concept that implementation of the surveillance guideline resulted in a change in practice. Thirty-one scans were performed on an outpatient basis (four before guideline and 27 after guideline implementation) and five were performed in the ER (all after guideline implementation). Correspondingly, the percentage of trauma service patients receiving duplex scans significantly increased from $9 \%$ (40/459) to $25 \%$ (133/539), following guideline implementation. To further ascertain whether this guideline resulted in a practice change, we examined the subset of patients admitted to the trauma service with a hospital length of stay greater than $2 \mathrm{~d}$, as the guideline was designed for patients with hospital length of stay greater than $2 \mathrm{~d}$. If the fraction of patients undergoing scanning following guideline implementation remained small, we would not have effected a practice change. As shown in Table 3, the percentage of trauma service patients with length of stay $>2 \mathrm{~d}$ undergoing duplex surveillance increased from 16\% (40/248) before guideline implementation to $43 \%$ (122/284) after guideline implementation. The number of patients receiving duplex scans in this group is slightly smaller than the number performed in all trauma service admissions because a few scans were done in the ER or were performed on an outpatient basis in patients who were discharged within two hospital days. We then retrospectively queried the trauma registry to see how many of the trauma service patients with hospital length of stay $>2 \mathrm{~d}$ would be classified as high-risk by our criteria (age $>55$, head injury with AIS $\geq 3$, spinal cord injury, complicated pelvic fracture, lower-extremity long bone fracture, major venous injury, multiple PRBC transfusions, or surgery longer than $2 \mathrm{~h}$ ), and how many of these underwent duplex scanning. However, there are limitations with the database in that any patient who had surgery (as opposed to surgery longer than $2 \mathrm{~h}$ ), any PRBC transfusion (as opposed to multiple blood transfusions), and any pelvic fracture (as opposed to complex pelvic fracture) were counted. Hence, the number of high-risk patients is overestimated. Noting this caveat, we found that in 2005-2006, 207 patients with hospital length of stay $>2 \mathrm{~d}$ would have met the surveillance criteria for high-risk; of these, 40 (19\%) patients underwent lower extremity duplex surveillance. In 2006-2007, 232 patients with length of stay $>2 \mathrm{~d}$ would have met these criteria; of these, 115 (49.6\%) went duplex surveillance.

Finally, there is the issue of cost. At our hospital, the cost of a bilateral lower extremity duplex scan in 2007 was approximately $\$ 800$; this does not include the professional interpretation fee, which was approximately $\$ 100$ additional. There were 199 more duplex scans performed on a trauma population with 80 additional patients, after the implementation of the routine surveillance guideline. If the rate of $9 \%$ duplex surveillance was continued for 998 patients, the cost would be $\$ 80,838$. If the rate of $25 \%$ duplex surveillance was continued for the 998 patients, the cost would increase to $\$ 224,550$ without a significant change in DVT detection rate.

\section{DISCUSSION}

We found a relatively low incidence of VTE disease in trauma patients. As noted by Adams et al., there is no consensus on the incidence of VTE events in trauma patients as well as on best method of prevention [1]. While a variety of injury patterns have been identified as high-risk for DVT development, there is controversy on the importance of various injuries. Cipolle et al. found that the vast majority of DVT occurred in patients with one of four major injury categories: closed head injury, long bone fracture, pelvic fracture, and 
spinal cord injury [14]. According to EAST practice management guidelines in 2002 , only spinal cord injury or spinal fractures were found to have level 1 data as being high-risk injuries for VTE development [7]. On meta-analysis, a variety of factors, including increasing ISS, blood transfusion, long bone fractures, pelvic fractures, and head injuries did not prove to be of major significance [7]. Meanwhile, a recent analysis of NTDB data suggested that age $\geq 40 \mathrm{y}$, extremity injury (AIS $\geq 3$ ), head injury (AIS $\geq 3$ ), ventilator days $\geq 3$, venous injury, and major surgery were important risk factors [10]. In our patients with VTE, the most common patient and injury characteristics, in descending order of frequency, were obesity, severe head injury, mechanical ventilation for 3 or more d, and surgery lasting longer than $2 \mathrm{~h}$.

Our study also further highlights the problem of infrageniculate DVT. Their diagnosis and management are controversial. In trauma patients with DVT, Cipolle et al. noted that $40 \%$ of DVT were located below the knee [14]. Iskander et al. found a $15.7 \%$ incidence of infrageniculate DVT in 698 screened trauma patients [13]. Furthermore, they found that $35.7 \%$ of patients with infrageniculate DVT had propagation of the clot; it propagated to a suprageniculate location about $33 \%$ of the time and to an infrageniculate location $66 \%$ of the time, despite thromboprophylaxis in a majority of patients. We noted a high incidence $(62 \%)$ of infrageniculate DVT in trauma patients. Furthermore, we noted that in three of the five patients with DVT and PE, the clots were infrageniculate in location.

While prophylaxis for DVT in trauma patients is generally advised, there is debate about the best agent for chemical prophylaxis and on the merits of mechanical prophylaxis in only high-risk trauma patients. A Cochrane review of general high-risk patients found that the addition of pharmacologic prophylaxis to mechanical compression devices significantly reduced the incidence of symptomatic pulmonary embolism (3\% versus $1 \%$ ) and DVT (4\% versus $1 \%$ ), as compared to compression devices alone [15]. Meanwhile, as compared with pharmacologic prophylaxis alone, the addition of compression devices significantly reduced the incidence of DVT ( $4.21 \%$ versus $0.65 \%$ ); however their studies were underpowered with regards to PE [15]. Meanwhile, a meta-analysis in 2010 of subcutaneous heparin versus mechanical compression prophylaxis in postoperative and trauma patients indicated a similar pooled relative risk for DVT and PE [16]. In this regard, Schwarcz et al. argued for the preferential use of low-molecular weight heparin, as opposed to unfractionated heparin in high-risk trauma patients $[17,18]$. In 2008, the ACCP recommended routine thromboprophylaxis for major trauma patients. As a grade $1 \mathrm{~A}$ recommendation, they suggested chemical prophylaxis with low molecular weight heparin [9]. As a grade $1 \mathrm{~B}$ recommendation, mechanical prophylaxis was advised when chemical prophylaxis is not warranted, or in combination with chemical prophylaxis [9]. The majority of trauma patients with VTE at our institution were receiving mechanical prophylaxis and/or chemical prophylaxis. An important reason for lack of thromboprophylaxis in patients with VTE was type of injury, i.e., chemical prophylaxis was withheld for variable periods of time in patients with significant head injury. We noted VTE events in patients on heparin or enoxaparin.

A recent survey by Haut et al. found a wide variation amongst trauma surgeons regarding the necessity for and timing of surveillance duplex scans in asymptomatic trauma patients [19]. Our finding of no significant change in the DVT detection rate after implementation of the surveillance guideline, despite a nearly 5 -fold increase in surveillance rate, contrast those of Haut et al., who noted that a 4-fold increase in duplex surveillance rate was associated with a 10 -fold increase in DVT diagnosis, after implementation of a practice management guideline in high-risk asymptomatic trauma patients [20].

An argument can be made for routine screening as previous work in medical-surgical ICU settings has demonstrated that the majority of ultrasound-proven DVT are asymptomatic [21]. In a prospective study, Crowther et al. demonstrated the futility of clinical examination in DVT detection in medical-surgical intensive care unit patients, with an area under curve of 0.57 to 0.59 depending on stratification/scoring system used [22]. In two additional studies by Cook et al., there was an unsuspected 9-10\% incidence of DVT in critically ill patients $[23,24]$. In a study of trauma patients, Adams et al. noted on routine surveillance imaging that began after seven days in nonambulatory or high-risk patients that 9/64 DVT identified were in symptomatic patients [1]. Similarly, Cipolle et al. in 2002 noted a yearly DVT rate of $0.7 \%-5 \%$ and a $\mathrm{PE}$ rate of $0.1 \%$ to $0.64 \%$ in trauma patients [14]. They noted that the positivity rate of duplex scanning with routine surveillance was $15 \%-20 \%$, where only $25 \%$ of DVT were symptomatic [14]. This is comparable to our finding that only $19 \%$ of DVT were symptomatic.

Furthermore, lower extremity screening for DVT is felt to be important because up to $90 \%$ of PE are thought to emanate from proximal lower extremity DVT and $25 \%-90 \%$ of DVT patients will develop post-phlebitic syndrome [6]. Additionally, the presence of DVT increases the risk for recurrent DVT by $10 \%$, and with more than one DVT, risk of recurrence increases to $20 \%$, even with anticoagulation [6]. Finally, an argument for evaluation of the asymptomatic leg in patients with unilateral lower extremity DVT is made by 
Pennell et al. who found a $20 \%$ incidence of contralateral leg vein DVT [25]. As such, earlier recognition and treatment of DVT may prevent some of these complications from developing.

Conversely, there are a variety of arguments against routine duplex surveillance. As noted above, the ACCP Consensus Conference in 2008 recommends screening only in high-risk patients who receive suboptimal or no thromboprophylaxis [9]. Even the argument that early detection and treatment of DVT would prevent subsequent pulmonary embolism has been challenged. To this end, in 2005, Borer et al. retrospectively evaluated the effect of screening with ultrasound and magnetic resonance venography on the incidence of pulmonary embolism in 973 patients with pelvic or acetabular fractures [3]. They found no significant difference in the incidence of pulmonary embolism in two time periods, before and after implementation of routine surveillance. Eight of the 10 patients who developed PE had undergone routine screening, which was negative for DVT. Similarly, Velmahos et al., in a retrospective study of 247 trauma patients undergoing CT pulmonary angiography and CT venography, identified pulmonary embolism in $19 \%$ of patients and DVT in $7 \%$ of patients [26]. Only $15 \%$ of patients with PE had a concomitant DVT [26]. Based on their work and several other studies cited, these authors suggested that many clots may originate de novo in the pulmonary circulation [26].

Given the above studies, which failed to demonstrate a reduction in $\mathrm{PE}$ rates with routine lower extremity surveillance, and given the substantial additional cost of routine surveillance without a significant change in DVT detection rate, our preliminary results further argue against routine surveillance.

This study has several limits. First, routine duplex surveillance was only done on lower extremities. As noted by Ascher et al. in a study of 210 patients with upper extremity or internal jugular vein DVT, $5.7 \%$ of patients were found to have PE that could be solely attributed to upper extremity DVT [27]. Additionally, while significantly more patients admitted to the trauma service following guideline initiation underwent surveillance duplex, not all patients admitted to the trauma service who would have met high-risk criteria received surveillance duplex scans. The lack of a higher guideline implementation rate may be in part because guideline implementation, while suggested, was not required. Finally, this study has the limitations of a retrospective review. As such, indications (e.g., symptoms) for duplex imaging were not always available. Furthermore, the ordering of the initial and subsequent surveillance duplex scans was variable. Finally, retrospective querying of the trauma registry for identification of high-risk patients encountered similar challenges.

\section{CONCLUSIONS}

We found that the incidence of VTE in patients admitted for injury was relatively low. Contrary to our expectation, routine lower extremity venous duplex surveillance in high-risk patients admitted to the trauma service did not result in significantly higher DVT detection rates despite a nearly 5 -fold increase in number of duplex scans. These preliminary data argue against the utilization of routine duplex surveillance in trauma patients, at a cost savings of about $\$ 900$ per patient. Further prospective study is needed to confirm this finding.

\section{ACKNOWLEDGMENTS}

The authors acknowledge Dr. David W. Mercer for his valuable suggestions in the preparation of the manuscript; they thank Deb Oltman and the Vascular Lab Staff at the Nebraska Medical Center for their hard-work and dedication.

\section{REFERENCES}

1. Adams RC, Hamrick M, Berenguer C, et al. Four years of an aggressive prophylaxis and screening protocol for venous thromboembolism in a large trauma population. J. Trauma 2008;65:300. discussion 306.

2. Huseynova K, Xiong W, Ray JG, et al. Venous thromboembolism as a marker of quality of care in trauma. J Am Coll Surg 2009; 208:547. 552.e1.

3. Borer DS, Starr AJ, Reinert CM, et al. The effect of screening for deep vein thrombosis on the prevalence of pulmonary embolism in patients with fractures of the pelvis or acetabulum: A review of 973 patients. J Orthop Trauma 2005;19:92.

4. Piotrowski JJ, Alexander JJ, Brandt CP, et al. Is deep vein thrombosis surveillance warranted in high-risk trauma patients? Am J Surg 1996;172:210.

5. Pierce CA, Haut ER, Kardooni S, et al. Surveillance bias and deep vein thrombosis in the national trauma data bank: The more we look, the more we find. J Trauma 2008;64:932. discussion 936.

6. Harris LM, Curl GR, Booth FV, et al. Screening for asymptomatic deep vein thrombosis in surgical intensive care patients. J Vasc Surg 1997;26:764.

7. Rogers FB, Cipolle MD, Velmahos G, et al. Practice management guidelines for the prevention of venous thromboembolism in trauma patients: The EAST practice management guidelines work group. J Trauma 2002;53:142.

8. Geerts WH, Pineo GF, Heit JA, et al. Prevention of venous thromboembolism: The Seventh ACCP Conference on Antithrombotic and Thrombolytic Therapy. Chest 2004;126:338S.

9. Hirsh J, Guyatt G, Albers GW, et al, American College of Chest Physicians Executive summary. American College of Chest Physicians Evidence-Based Clinical Practice Guidelines (8th edition). Chest 2008;133:71S.

10. Haut ER, Chang DC, Pierce CA, et al. Predictors of posttraumatic deep vein thrombosis (DVT): Hospital practice versus patient factors-an analysis of the NationalTrauma Data Bank (NTDB). J Trauma 2009;66:994. discussion 999. 
11. Knudson MM, Ikossi DG, Khaw L, et al. Thromboembolism after trauma: An analysis of 1602 episodes from the American College of Surgeons National Trauma Data Bank. Ann Surg 2004; 240:490. discussion 496.

12. Gearhart MM, Luchette FA, Proctor MC, et al. The risk assessment profile score identifies trauma patients at risk for deep vein thrombosis. Surgery 2000;128:631.

13. Iskander GA, Nelson RS, Morehouse DL, et al. Incidence and propagation of infrageniculate deep venous thrombosis in trauma patients. J Trauma 2006;61:695.

14. Cipolle MD, Wojcik R, Seislove E, et al. The role of surveillance duplex scanning in preventing venous thromboembolism in trauma patients. J Trauma 2002;52:453.

15. Kakkos SK, Caprini JA, Geroulakos G, et al. Combined intermittent pneumatic leg compression and pharmacological prophylaxis for prevention of venous thromboembolism in high-risk patients. Cochrane Database Syst Rev 2008;4:CD005258.

16. Eppsteiner RW, Shin JJ, Johnson J, et al. Mechanical compression versus subcutaneous heparin therapy in postoperative and post-trauma patients: A systematic review and meta-analysis. World J Surg 2010;34:10.

17. Schwarcz TH, Quick RC, Minion DJ, et al. Enoxaparin treatment in high-risk trauma patients limits the utility of surveillance venous duplex scanning. J Vasc Surg 2001; 34:447.

18. Schwarcz TH, Matthews MR, Hartford JM, et al. Surveillance venous duplex is not clinically useful after total joint arthroplasty when effective deep venous thrombosis prophylaxis is used. Ann Vasc Surg 2004;18:193.

19. Haut ER, Patel A, Haider AH, et al. Duplex ultrasound screening for deep vein thrombosis (DVT) in asymptomatic trauma patients: Survey results of current opinions and practice patterns from 316 trauma surgeons. J Surg Res 2010;158:287.

20. Haut ER, Noll K, Efron DT, et al. Can increased incidence of deep vein thrombosis (DVT) be used as a marker of quality of care in the absence of standardized screening? The potential effect of surveillance bias on reported DVT rates after trauma. J Trauma 2007;63:1132. discussion 1135.

21. Cook DJ, Crowther MA. Thromboprophylaxis in the intensive care unit: Focus on medical-surgical patients. Crit Care Med 2010;38:S76.

22. Crowther MA, Cook DJ, Griffith LE, et al. Deep venous thrombosis: Clinically silent in the intensive care unit. J Crit Care 2005; 20:334.

23. Cook DJ, Rocker G, Meade M, et al. PROTECT Investigators, and Canadian Critical Care Trials Group Prophylaxis of Thromboembolism in Critical Care (PROTECT) Trial: A pilot study. J Crit Care 2005;20:364.

24. Cook D, Douketis J, Meade M, et al, Canadian Critical Care Trials Group. Venous thromboembolism and bleeding in critically ill patients with severe renal insufficiency receiving dalteparin thromboprophylaxis: Prevalence, incidence, and risk factors. Crit Care 2008;12:R32.

25. Pennell RC, Mantese VA, Westfall SG. Duplex scan for deep vein thrombosis-defining who needs an examination of the contralateral asymptomatic leg. J Vasc Surg 2008;48:413.

26. Velmahos GC, Spaniolas K, Tabbara M, et al. Pulmonary embolism and deep venous thrombosis in trauma: Are they related? Arch Surg 2009;144:928.

27. Ascher E, Salles-Cunha S, Hingorani A. Morbidity and mortality associated with internal jugular vein thromboses. Vasc Endovascular Surg 2005;39:335. 